\title{
Surgical Resection of Malignant Lymphoma in the Right Atrium after Systemic Chemotherapy
}

\author{
Takashi IgAwA, Koji NAGAFuJI, Junichi EJIMA*, Kazuta NAKASUgA, Hiroyuki ITO, \\ Yoshikazu KAJI, Toshihiro MiYAmOTO and Mine HARADA
}

\begin{abstract}
A 74-year-old man was referred to us for evaluation of a tumor in the right atrium (RA). Transesophageal echocardiography (TEE) showed an unmovable $50 \times 60 \mathrm{~mm}$ mass in the RA. Based on histological findings of subcutaneous tumors in the right abdominal wall, he was diagnosed as malignant lymphoma (ML), and treated with a THP-COP regimen. Upon completion of first THO-COP therapy, TEE showed marked regression of the mass and division into 3 masses, one of which showed marked floating movement with a small stalk. To prevent the risk of embolic events, surgical resection was performed. Resected tumors were necrotic tissues. Serial imaging of cardiac tumor and surgical resection is desirable to decrease the possibility of embolic complication.

(Internal Medicine 42: 336-339, 2003)
\end{abstract}

Key words: cardiac tumor, surgery, operation, thromboembolism

\section{Introduction}

Cardiac involvement as an initial presentation of malignant lymphoma (ML) is rare and represents $1 \%$ of all primary cardiac tumors (1). ML has been reported to constitute over $17 \%$ of the total metastases by malignant neoplasms to the heart, and up to $20 \%$ of patients dying from lymphomas are found to have cardiac involvement (2-4). Since biopsy of tumors in the heart is difficult and histological proof is mandatory, the diagnosis of ML in the heart is challenging. Since ML is chemoradio-sensitive and potentially curable (5), early and correct diagnosis is desired. One of the complications of ML in the heart is thromboembolism, which is sometimes fatal (6-10).

Here, we report a patient with ML involving the right atrium (RA). The tumor in RA showed floating movement after systemic chemotherapy, and was successfully resected to prevent thromboembolic complications.

\section{Case report}

A 74-year-old man developed fever and exertional dyspnea in November 2001. He was admitted to the regional hospital on November 23, 2001; right pulmonary nodule, pericardial effusion, and paroxysmal atrial fibrillation were pointed out. Transbronchial lung biopsy was not diagnostic. In December 2001, chest computed tomography (CT) scan and transesophageal echocardiography (TEE) revealed a tumor in the right atrium (RA). He was referred to us on January 21,2002 . He had $5 \mathrm{~kg}$ of body weight loss in 6 months. On examination, his temperature was $38.1^{\circ} \mathrm{C}$, blood pressure $110 / 62 \mathrm{mmHg}$, pulse rate 110 beats/min. Heart sound was normal and there was wheezing in both lung fields. Superficial lymph nodes were not palpable. Blood examination revealed increased levels of lactate dehydrogenase (LDH, 709 IU/l, normal range 119-229) and soluble IL-2 receptor (sIL-2R, 3,640 U/ml, normal range 220-530). A chest $\mathrm{X}$-ray demonstrated mild cardiomegaly and a mass lesion in the right middle lung field. Electrocardiography showed sinus tachycardia and complete right bundle branch block. CT scan showed a mass of $60 \mathrm{~mm}$-diameter in RA extending to the superior vena cava, and a tumor of $25 \times 30 \mathrm{~mm}$ in size in the right middle lobe (Fig. 1). Gallium (67Ga) scintigraphy showed positive uptake in the heart and upper abdomen. TEE demonstrated an unmovable mass of $50 \times 60 \mathrm{~mm}$ in the free wall of RA (Fig. 2A). Transbronchial lung biopsy of the mass in right lung showed old inflammatory change suggesting inflammatory pseudo-tumor. On February 5, obstructive jaundice (total bilirubin $2.6 \mathrm{mg} / \mathrm{dl}$ ) developed and

From the First Department of Internal Medicine, Faculty of Medicine, Kyushu University Hospital, Fukuoka and *Department of Cardiology, Fukuoka Higashi Hospital, Fukuoka

Received for publication August 15, 2002; Accepted for publication January 22, 2003

Reprint requests should be addressed to Dr. Koji Nagafuji, the First Department of Internal Medicine, Faculty of Medicine, Kyushu University Hospital, 3-1-1 Maidashi, Higashi-ku, Fukuoka 812-8582 


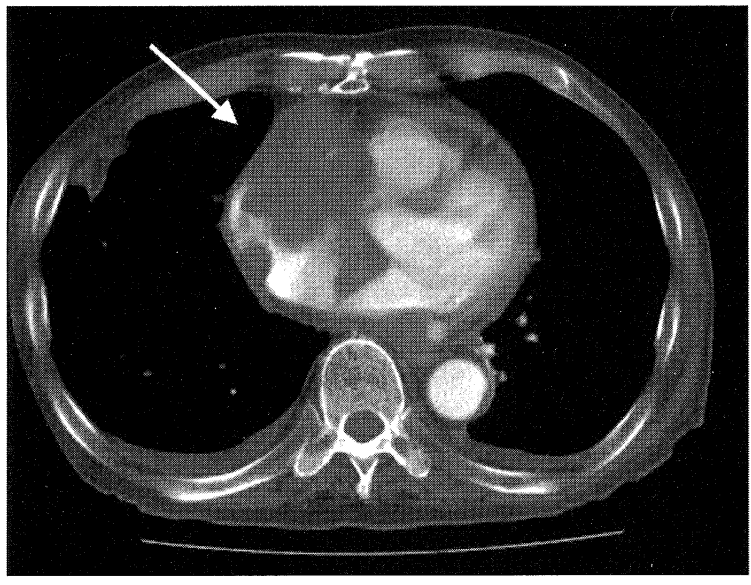

A

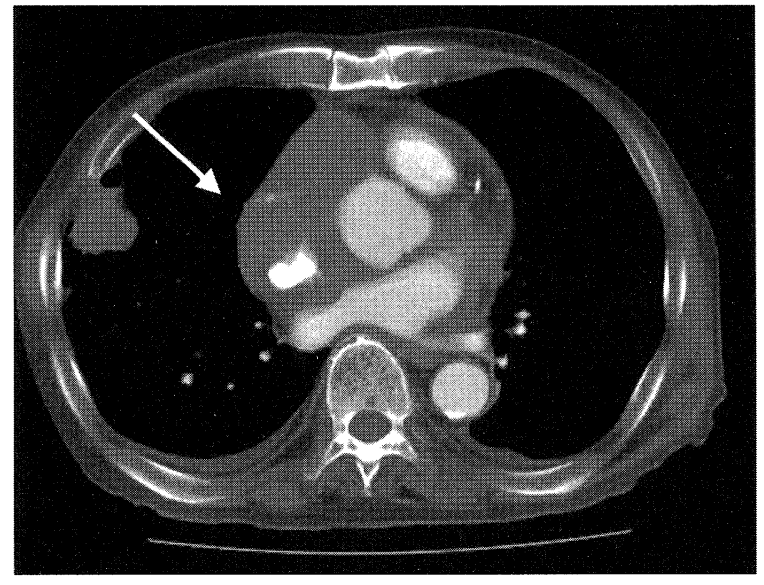

$\mathrm{B}$

Figure 1. Computed tomography (CT) scan demonstrated a mass (arrow) occupying the right atrium (A) extending to the superior vena cava $(B)$.

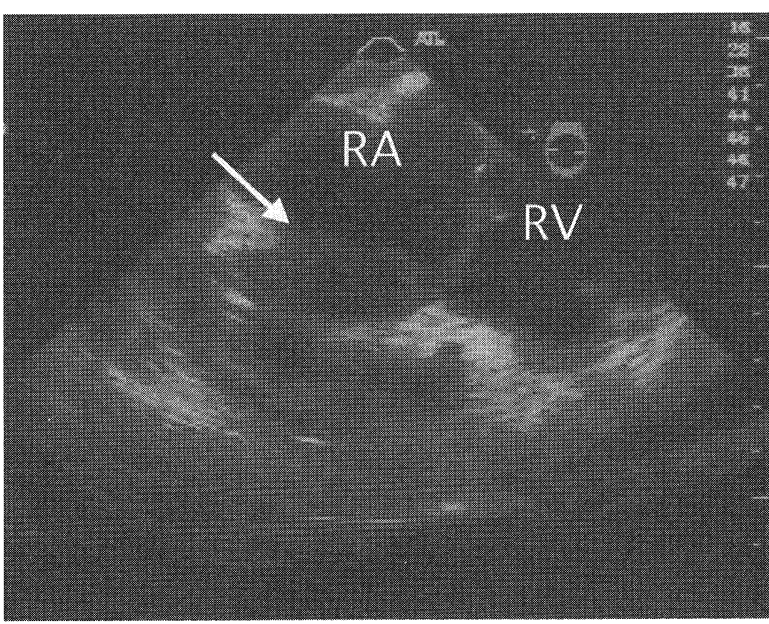

A

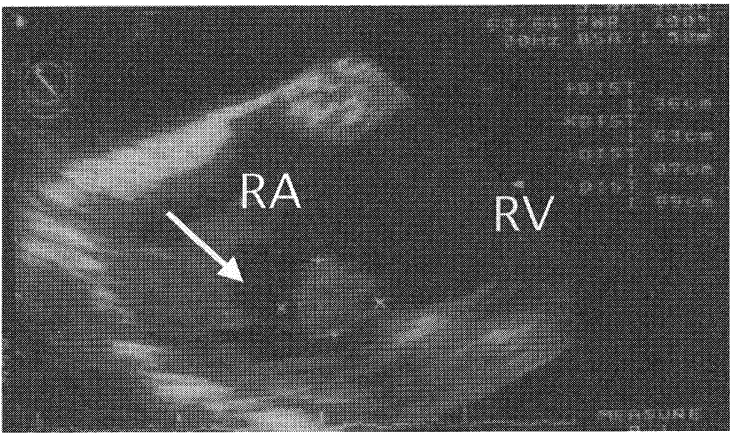

$\mathrm{B}$

Figure 2. Transesophageal echocardiography (TEE) showed a large mass (arrow) in the right atrium (RA) (A). After systemic chemotherapy for malignant lymphoma, TEE showed regression of the mass in RA resulting in 3 masses, one of which was floating pedunculated mass on a stalk suggesting the risk of emboli (B).

abdominal CT scan revealed a $3 \mathrm{~cm}$-diameter mass in the pancreas head with biliary tract dilatation, and subcutaneous tumors in the right abdominal wall. A drainage tube was inserted into the biliary tract endoscopically to relieve jaundice, and biopsy of the subcutaneous mass was done. Pathological examination showed diffuse proliferation of atypical large lymphoid cells, which were positive for B cell markers (CD10, CD19, CD20), but negative for T cell markers (CD2, CD3). Bone marrow aspiration at the sternum showed no malignant cells. Based on these findings, a diagnosis of ML diffuse large B cell type, stage IVB involving heart, pancreas, and right abdominal wall was made; international prognostic index was high risk. On February 21, a THP-COP regimen consisting of pinorubicin $45 \mathrm{mg} / \mathrm{m}^{2}$, cyclophosphamide $750 \mathrm{mg} / \mathrm{m}^{2}$, vincristine $2 \mathrm{mg} /$ body, and prednisolone $100 \mathrm{mg} /$ body for 5 days was started. Prompt fever lysis and marked improvement of constitutional symptoms were obtained. The level of LDH returned to normal. His subcutaneous masses disappeared. Upon the completion of first THO-COP therapy, TEE showed marked regression of the mass in RA and the division into 3 masses, one of which showed floating pedunculated mass on a stalk suggesting a risk of embolism. The floating mass was about 1.5 $\mathrm{cm}$ in diameter (Fig. 2B), and if it were to float to the pulmonary artery, it could cause hemodynamic collapse. To prevent embolic events, surgical resection of the masses in RA with cardiopulmonary bypass was done on March 11 . Three tumors in RA were resected successfully. Histological 


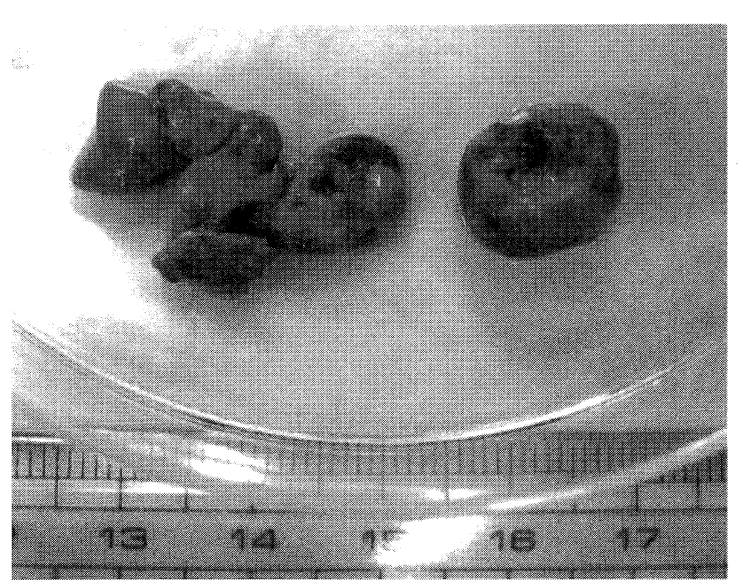

A

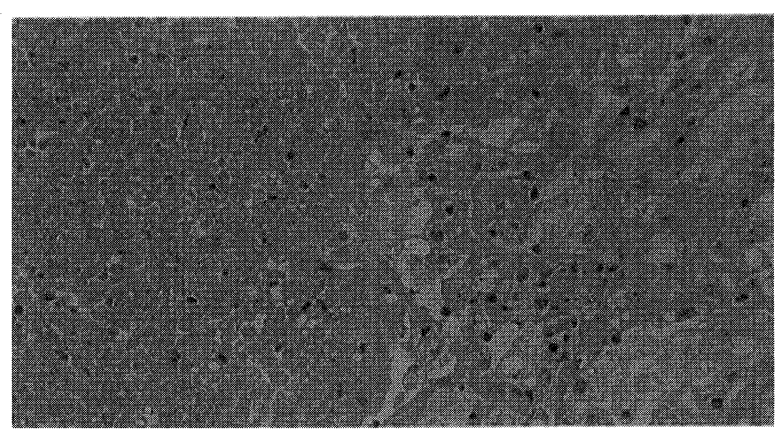

$\mathrm{B}$

Figure 3. Resected tumors from the right atrium. (A) Macroscopic appearance. (B) Histological examination showed massive necrosis with foamy macrophages (HE stain, $\times 100$ ).

examination showed massive necrosis with foamy macrophages (Fig. 3). His post-operative course was uneventful. On post-operative day 14 , the second THO-COP therapy was given. After the 3rd cycle of THO-COP, CT scan demonstrated disappearance of the mass in the pancreas head, and the biliary drainage tube was removed.

\section{Discussion}

The treatment of ML involving the heart has not been established $(11,12)$.

There are two types of cardiac involvement by ML. The first type, primary cardiac ML, by definition is extranodal lymphoma findings in the heart and/or pericardium only (1). Most primary cardiac ML are reported to be B-cell lineage (13). The second is ML metastasis to the heart. With the progress of imaging techniques including echocardiography, $\mathrm{CT}$, and magnetic resonance imaging, the detection of a mass in the heart become easier. However, for the diagnosis of etiology, histological or cytological examination are mandatory. For the biopsy of an intracardiac mass, transcatheter biopsy or open heart surgery is necessary. If pericardial effusion is massive, cytological examination sometime leads to correct diagnosis $(11,12)$.

In the present case, it was difficult to locate the primary site of ML. However, the first-recognized lesion was his heart with pericardial effusion, and his ML was B cell lineage. Thus, the primary site might be the heart. When histological examination of his abdominal subcutaneous mass lead to the diagnosis of ML, ML was extended to the heart, pancreas, and right abdominal wall. Since systemic chemotherapy with THP-COP induced the prompt regression of heart and pancreas lesions, the diagnosis of ML was confirmed. However, the regression caused the RA tumor from the wall to float with a stalk attached. Serial echocardiography revealed increasing movability, and we decided to remove the floating tumors to prevent the risk of embolic events. The resected tumors were necrotic tissues, and there was a low possibility of subsequent chemotherapy to further regress RA tumors. Several reports have described fatal or near-fatal embolic episodes in patients with cardiac ML (610). In all of those case reports, the embolism occurred before the initiation of systemic chemotherapy. In contrast, effective chemotherapy made the RA tumor small and frail in the present case. Early and correct diagnosis of ML, systemic chemotherapy, and serial examination of the heart by echocardiography are necessary for the proper management of cardiac ML.

In conclusion, we report a patient with ML involving the heart. Correct diagnosis and serial imagings of the heart made systemic chemotherapy and surgical resection possible. Since ML is a potentially curable disease, we should consider the possibility of ML when a cardiac mass is found.

\section{References}

1) McAllister HA, Fenoglio JJ. Tumors of the cardiovascular system. Atlas of tumor pathology. 2nd series, Fasicle 15. Armed Forces Institute of Pathology. Washington, DC, 1978: 99-100

2) Roberts WC, Glancy DL, DeVita VT Jr. Heart in malignant lymphoma (Hodgkin's disease, lymphosarcoma, reticulum cell sarcoma and mycosis fungoides). A study of 196 autopsy cases. Am J Cardiol 22: 85107, 1968.

3) McDonnell PJ, Mann RB, Bulkley BH. Involvement of the heart by malignant lymphoma: a clinicopathologic study. Cancer 49: 944-951, 1982.

4) Lam KY, Dickens P, Chan AC. Tumors of the heart. A 20-year experience with a review of 12,485 consecutive autopsies. Arch Pathol Lab Med 117: 1027-1031, 1993

5) Kouroukis CT, Browman GP, Esmail R, Meyer RM. Chemotherapy for older patients with newly diagnosed, advanced-stage, aggressivehistology non-Hodgkin lymphoma: a systematic review. Ann Intern Med 136: 136-143, 2002.

6) Bestetti RB, Soares FA, Soares EG, Oliveira JS. Primary lymphoma of the right atrium with fatal neoplastic pulmonary embolism. Am Heart 
J 124: 1088-1090, 1992.

7) Serrano M, Iglesias A, Bellas C, Espino R. Left atrial ball thrombus with histologic features of extranodal B-cell lymphoma--prolonged survival after surgery. Acta Oncol. 33: 575-576, 1994.

8) Tanaka T, Sato T, Akifuji Y, et al. Aggressive non-Hodgkin's lymphoma with massive involvement of the right ventricle. Intern Med 35: 826-830, 1996.

9) Skalidis EI, Parthenakis FI, Zacharis EA, et al. Pulmonary tumor embolism from primary cardiac B-cell lymphoma. Chest 116: 1489-1490, 1999.

10) Inoue $\mathrm{H}$, Shimokawa $\mathrm{S}$, Iguro $\mathrm{Y}$, et al. Involvement of the right atrium by malignant lymphoma as a cause of right cardiac failure: report of a case. Surg Today 30: 394-396, 2000.

11) Petersen CD, Robinson WA, Kurnick JE. Involvement of the heart and pericardium in the malignant lymphomas. Am J Med Sci 272: 161165, 1976

12) Ceresoli GL, Ferreri AJ, Bucci E, et al. Primary cardiac lymphoma in immunocompetent patients: diagnostic and therapeutic management. Cancer 80: 1497-1506, 1997.

13) Curtsinger CR, Wilson MJ, Yoneda K. Primary cardiac lymphoma. Cancer 64: 521-525, 1989. 